\title{
In situ characterization technique for nanotribological investigations
}

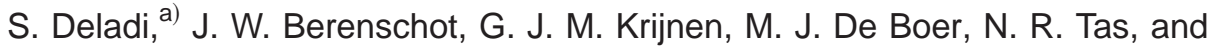 \\ M. C. Elwenspoek \\ MESA + Research Institute, University of Twente/Transducers Science and Technology, P.O. Box 217, \\ 7500 AE Enschede, The Netherlands
}

(Received 2 April 2004; accepted 30 September 2004; published online 23 December 2004)

\begin{abstract}
An innovative technique has been developed to enable in situ monitoring of mechanical surface modification. The method is based on using a test surface and a sharp tip located on two different cantilevers; one for mechanical surface modification and the other for in situ detection of surface topography change. The device can be used in standard atomic force microscope, the image obtained during scanning contains information about a partly modified and a partly unmodified topography for each scanning line, thus quantification of surface topography modification (e.g., wear) or the change of different parameters (e.g., friction force) can be followed as it occurs. The characterization technique and typical results are presented. (c) 2005 American Institute of Physics. [DOI: 10.1063/1.1834712]
\end{abstract}

Scanning probe microscopy (SPM) is a very important technique in surface science. Here we concentrate on the use of SPM for the study of in situ surface modifications. Recent developments enable nanolithography and nanopatterning, ${ }^{1,2}$ however the main utilization relies on characterization of surfaces, ${ }^{3}$ determination of material properties, ${ }^{4-6}$ measurement of mechanical properties of micro/nanoscale structures. ${ }^{7}$ Besides, there is increased interest in using the same tool simultaneously for different purposes, which is a driving force in the development of multicantilevered probes. ${ }^{8,9}$

An important member of the SPM family is the atomic force microscope (AFM). Since the information on which the images are based contains aspects of the interaction between the scanning tip and the scanned surface, the AFM has extensively been used for tribological investigations including adhesion, friction, and wear of various materials for different loading conditions with or without lubrication. ${ }^{10-14}$ State of the art nanoscale wear studies ${ }^{12-14}$ use the same tip for wearing and measuring. Wearing is done at a high load while subsequent imaging is done with the same probe on a larger area applying a smaller load. Since zoom-out and change of load has to be made frequently, the contact conditions are in practice changed each time the worn region is examined.

The in situ technique presented provides information about modified and unmodified topographies at the same time, and allows a wider range of possibilities for surface modification without compromising image quality.

The tool (wear-AFM probe) developed for this purpose has the following advantages: (i) it can be used in standard AFM equipment, (ii) because it contains a regular AFM tip, subnanometer change of surface topography can be detected, (iii) in situ adhesion, friction, and wear investigations are facilitated for a wide range of loading conditions for various material-couples.

\footnotetext{
a)Electronic mail: s.deladi@ewi.utwente.nl
}

The wear-AFM probe consists of two cantilevers separated by a given distance that allows to a certain extent overlap of the scanning ranges of the two tips (Fig. 1): a test surface located on a so-called multifunctional cantilever which generates the change of the surface topography within its scanning range (rubbing range), and a sharp tip placed on another so called detection cantilever for in situ monitoring of the surface topography within its scanning range. The minimum separation distance between the cantilevers is limited by the diameter of the AFM laser beam that is focused only on the tip of the detection cantilever, assuring that any movement of the multifunctional cantilever does not influence the reflection of the laser beam onto the photodiodes. The scanning area of the detection tip determines the size of the images captured during measurements. They consist of a part where little or no wear occurs (due to very low loading of the detection tip against the surface) and a part where wear is generated, defined by the overlap between the scanning and the rubbing ranges. The ratio of the normal loads as exerted by the multifunctional cantilever and the detection tip is determined by the geometrical characteristics (angle, height, width, thickness) and material properties of the cantilevers.

The fabrication process of the device is based on the fabrication of a single-cantilever AFM probe ${ }^{15}$ with additional techniques for specific functionalities. On the topside of the cantilevers a thin Au layer is deposited to enhance reflection of the laser beam to the photodiode, and then the rubbing surface can be coated optionally with a chosen material (e.g., Cr) by shadow-masking technique. A fabricated wear-AFM probe is shown in Fig. 2, this particular configuration being used for the experiments presented.

This work is restricted to demonstrate typical results of the measurement technique at the nanoscale.

Due to the inclined mounting of the device in an AFM it is possible to exert dissimilar normal forces on a sample surface with the two cantilevers. Larger force can be applied with the multifunctional cantilever than with the detection cantilever for the configuration shown in Fig. 1. The test 


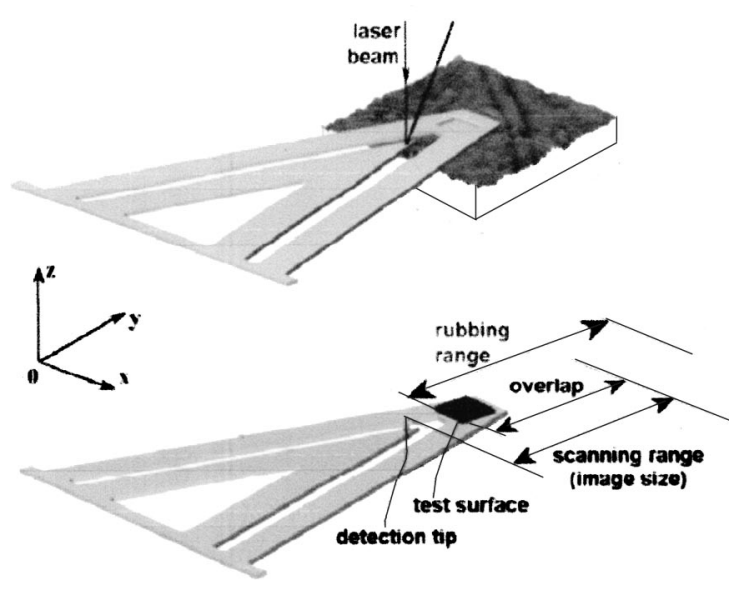

FIG. 1. Sketch of the working principle of the device used for in situ characterization of surface modification; top and bottom views, respectively.

surface is much larger than the detection tip, thus the pressure exerted by the tip can be larger than that applied by the test surface in case the latter is entirely in contact with the sample. However, due to the inclined mounting of the probe the friction force that appears between the sample and the test surface during scanning will cause a torque that rotates the test surface, making the contact occur along the leading edge. Scanning a sample with a rectangular shaped surface instead of a sharp tip has proved this principle, the asperities encountered appeared as ridges with length approximately equal to the edge of the test surface. The smaller the test surface the larger the applied contact pressure, but it is possible to further reduce the contact edge by using circular test surface instead of rectangular one. In these conditions the pressure exerted by the edge of the test surface is larger than that applied on the detection tip.

A Si wafer roughened by reactive ion etching $\left(R_{a}\right.$ $\sim 14.5 \mathrm{~nm}$ ) has been used as a sample for in situ wear experiment. The detection tip was loaded with $25 \mathrm{nN}$ against the sample, which according to the calculations ${ }^{16}$ based on the geometrical characteristics of the multifunctional cantilever and the properties of the material the cantilever is made of $\left(\mathrm{Si}_{x} \mathrm{~N}_{y}\right.$ with Young modulus $\left.300 \mathrm{GPa}\right)$, resulted in $0.8 \mu \mathrm{N}$ loading of the circular test surface with a radius of $4 \mu \mathrm{m}$ against the sample. In Fig. 3 we show the results of repeated scanning of a region. It can be observed that the modification where only the detection tip scans is very small compared to that in the overlap region, where also the rubbing surface

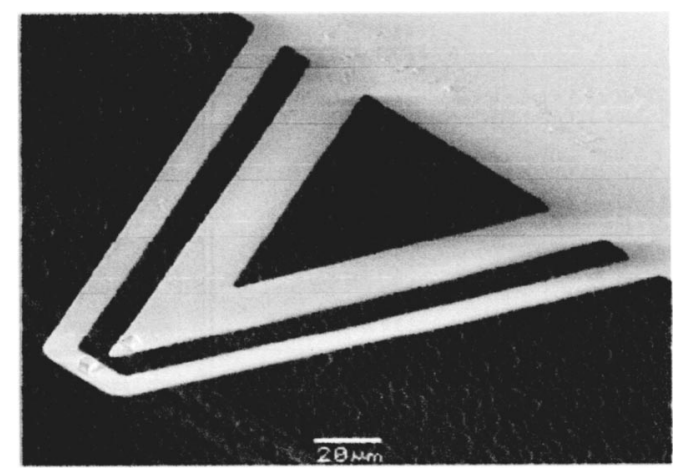

FIG. 2. Bottom view of a fabricated wear-AFM probe on a tilted SEM table.

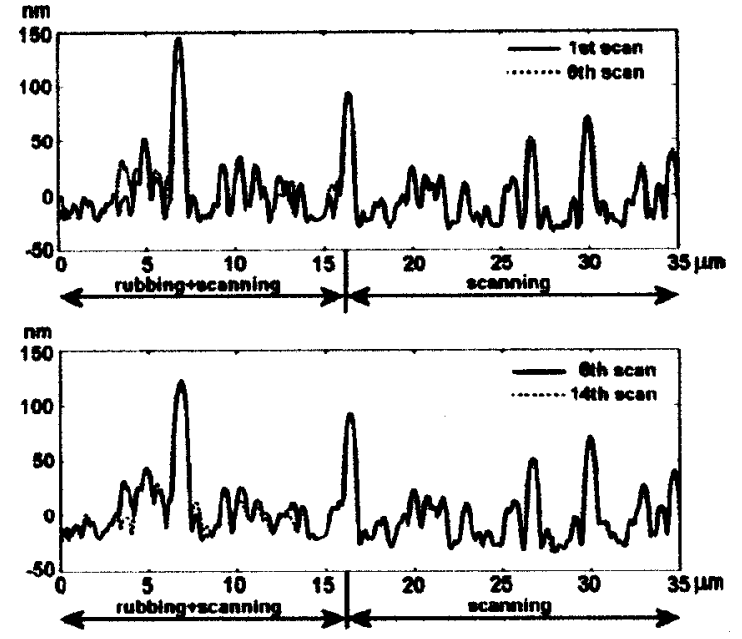

FIG. 3. Comparison of the profiles for the first, sixth, and fourteenth scan cycles for the same section of the Si sample. There is slight topography change where just the detection tip scans, but in the overlap region the modification is significant.

operates. Since the loading of the detection tip can be minimized, for few scanning cycles the topography change in the scanning region can almost be neglected. In the overlap region the topography modification is significant and the comparisons between successively taken images show that the material is continuously redistributed.

Another powerful in situ wear example is when the slow scan axis is disabled and scanning occurs along a single line. The image is built up from topography (or friction) information of subsequent scan cycles. A smooth $\mathrm{Si}$ surface $\left(R_{a}\right.$ $\sim 0.4 \mathrm{~nm}$ ) was chosen as a sample and the test surface on the wear-AFM probe was square shaped with $5 \mu \mathrm{m}$ sides. The load on the detection tip was optimized so that the surface is not damaged due to scanning with the tip, while the force applied on the edge of the square test surface was $0.4 \mu \mathrm{N}$. In Fig. 4 it is shown that the depth of the wear track increases from $0.2 \mathrm{~nm}$ in the first scan to $1.3 \mathrm{~nm}$ for the 256th scan cycle. The $0.2 \mathrm{~nm}$ deep track after the first scan cycle is attributed to removal of native oxide that forms on Si surfaces and possible bulk deformation.

An important test for thin-film coated materials is the determination of critical load/wear history for film removal. For this experiment a $\mathrm{Si}$ wafer was coated with $5 \mathrm{~nm}$ thick Pt, then the sample was subjected to in situ wear test. In Fig. 5(a) the topography of a $40 \times 40 \mu \mathrm{m}^{2}$ area is shown after one

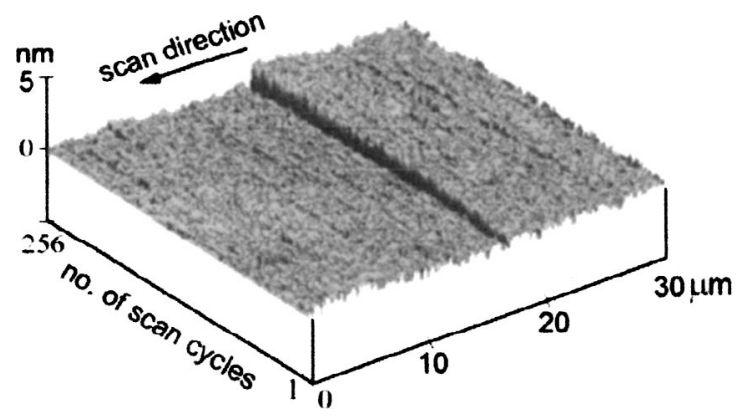

FIG. 4. In situ wear test on a smooth $\left(R_{a} \sim 0.4 \mathrm{~nm}\right) \mathrm{Si}$ surface. The image is built up from subsequent scanning along a single line (slow scan axis disabled). The depth of the wear track increases from $0.2 \mathrm{~nm}$ (first scan) to $1.3 \mathrm{~nm}$ (256th scan) as the scanning proceeds. 

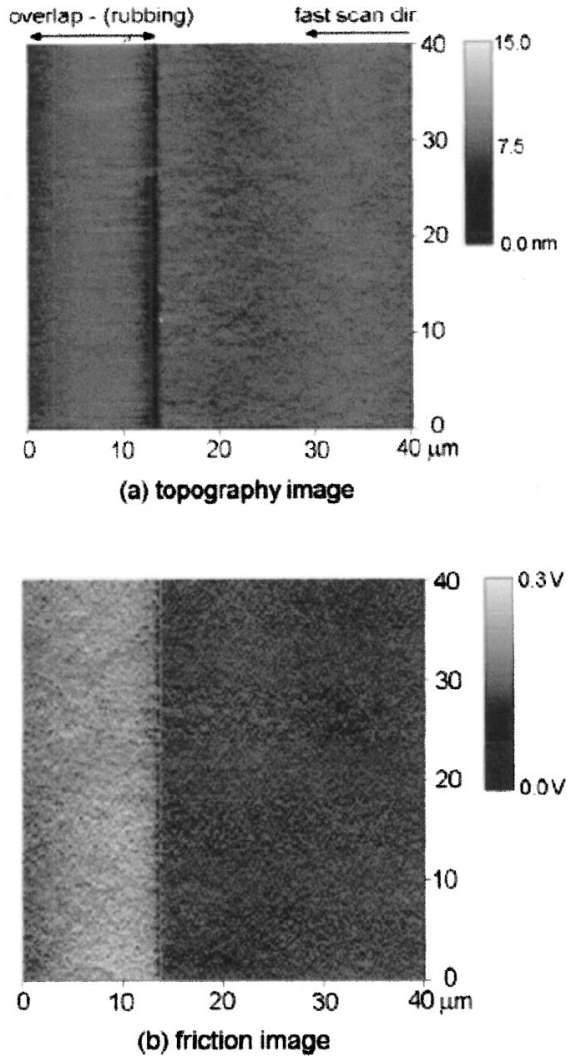

FIG. 5. In situ wear and friction measurement on a Pt coated Si wafer. (a) Topography image of a $40 \times 40 \mu \mathrm{m}^{2}$ area in which wear due to rubbing in the overlap area can be observed; (b) friction signal of the same area obtained simultaneously with the topography image, which shows that the $5 \mathrm{~nm}$ thick Pt coating is worn because the friction signal has completely modified in the overlap region.

complete scan cycle, the region where the rubbing surface operates being modified. Figure 5(b) shows the in situ friction signal image in which two regions can be distinguished where the friction signal is uniform. The region where the friction signal changed corresponds to the region where rubbing occurred and the topography has been modified. The friction signal suggests that the Pt film is worn away, which is confirmed by the step height of $5.1 \mathrm{~nm}$ in the topography image. The effect of the surface modification on adhesion strength (pull off force) can also be investigated in situ at any place within the scanning range.

In principle, the multifunctional cantilever can be positioned either in front or behind the detection cantilever by adjusting the ratio between the heights of the tip/surface and the geometrical characteristics of the cantilevers. The option for more than two cantilevers for adding extra functionalities is not excluded but is not practical, because the overlap region of scanning ranges of the tips and/or surfaces is diminished, and it may be difficult to control the applied normal load on the tips/surfaces separately using a standard AFM.

The multifunctional cantilever can perform other functions than wearing the surfaces. It is possible to incorporate a heater, ${ }^{6}$ which would enable investigation of the wear processes at elevated temperatures for various material couples. Improvement of the force control on the test surface can be made using active multifunctional cantilever, ${ }^{9}$ which allows controlling the contact force in a wide range and not only predefined by the properties of materials and the geometry.

We presented a technique that enlarges nanoscale investigation of mechanical surface modification and we demonstrated new characterization capabilities. The tool extends the possibilities of nanoscale tribological investigations with standard AFM equipment, allowing in situ detection of topographic surface modifications as generated in a controlled manner by a tip or surface located on the multifunctional cantilever. Surface modification with subnanometer vertical resolution can be detected due to in situ comparison between modified and unmodified regions contained in the same image. This is important for fundamental understanding of wear and for reliability studies of micro/nanoelectromechanical systems.

The authors are thankful for the financial support of the Dutch Technology Foundation (STW).

${ }^{1}$ R. D. Piner et al., Science 283, 661 (1999).

${ }^{2}$ L. Fu et al., Nano Lett. 3, 757 (2003).

${ }^{3}$ B. Bhushan, Micro/Nano Tribology (CRC Press, Boca Raton, 1999), ISBN 0-8493-8402-8.

${ }^{4}$ A. R. Vaz et al., in Proc. Nanotech 2003, Vol. 3, p. 177 (2003).

${ }^{5}$ A. J. Brook et al., J. Micromech. Microeng. 13, 124 (2003).

${ }^{6}$ O. Nakabeppu and T. Suzuki, J. Therm. Anal. 69, 727 (2002).

${ }^{7}$ S. Sundararajan and B. Bhushan, Sens. Actuators, A 101, 338 (2002).

${ }^{8}$ S. M. Altman and J. K. H. Horber, U.S. Patent No. 9583411 B1 (2003).

${ }^{9}$ D. Bullen et al., Appl. Phys. Lett. 84, 789 (2004).

${ }^{10}$ B. Bhushan et al., Nature (London) 374, 607 (1995).

${ }^{11}$ S. Sundararajan and B. Bhushan, J. Vac. Sci. Technol. A 19, 1777 (2001).

${ }^{12}$ H. Liu et al., J. Vac. Sci. Technol. A 19, 1234 (2001).

${ }^{13}$ V. N. Koinkar and B. Bhushan, Sens. Actuators, A 57, 91 (1996).

${ }^{14}$ V. N. Koinkar and B. Bhushan, J. Vac. Sci. Technol. A 14, 2378 (1996).

${ }^{15}$ T. A. Albrecht et al., J. Vac. Sci. Technol. A 8, 3386 (1990).

${ }^{16}$ J. E. Sader, Rev. Sci. Instrum. 66, 4583 (1995). 